\begin{tabular}{|c|l|}
\hline Title & $\begin{array}{l}\text { Majorana fermions and odd-frequency Cooper pairs in a normal-metal nanowire proximity-coupled to a topological } \\
\text { superconductor }\end{array}$ \\
\hline Author(s) & A sano, Y asuhiro; Tanaka, Yukio \\
\hline Citation & $\begin{array}{l}\text { Physical Review B, 87(10), 104513 } \\
\text { https://doi.org/10.1103/PhysRevB.87.104513 }\end{array}$ \\
\hline Issue Date & 2013-03-15 \\
\hline Doc URL & http://hdl.handle.net/2115/52640 \\
\hline Rights & @2013A merican Physical Society \\
\hline Type & article \\
\hline File Information & PhysRevB.87.104513.pdf \\
\hline
\end{tabular}

Instructions for use 


\title{
Majorana fermions and odd-frequency Cooper pairs in a normal-metal nanowire proximity-coupled to a topological superconductor
}

\author{
Yasuhiro Asano ${ }^{1}$ and Yukio Tanaka ${ }^{2}$ \\ ${ }^{1}$ Department of Applied Physics and Center for Topological Science \& Technology, Hokkaido University, Sapporo 060-8628, Japan \\ ${ }^{2}$ Department of Applied Physics, Nagoya University, Nagoya 464-8603, Japan
}

(Received 18 April 2012; revised manuscript received 4 February 2013; published 15 March 2013)

\begin{abstract}
We discuss a strong relationship between Majorana fermions and odd-frequency Cooper pairs which appear at a disordered normal $(\mathrm{N})$ nanowire attached to a topologically nontrivial superconducting $(\mathrm{S})$ one. The transport properties in superconducting nanowire junctions show universal behaviors irrespective of the degree of disorder: the quantized zero-bias differential conductance at $2 e^{2} / h$ in NS junction and the fractional current-phase $(J-\varphi)$ relationship of the Josephson effect in SNS junction $J \propto \sin (\varphi / 2)$. Such behaviors are exactly the same as those found in the anomalous proximity effect of odd-parity spin-triplet superconductors. We show that the odd-frequency pairs exist wherever the Majorana fermions stay.
\end{abstract}

DOI: 10.1103/PhysRevB.87.104513

PACS number(s): 74.81.Fa, 74.25.F-, 74.45.+c

\section{INTRODUCTION}

The Majorana fermion (MF) satisfying a special relation of $\gamma=\gamma^{\dagger}$ with $\gamma\left(\gamma^{\dagger}\right)$ being the annihilation (creation) operator has been an exciting object since the original prediction by Majorana. ${ }^{1}$ Finding MFs and controlling of Majorana bound states (MBSs) are hot research issues in condensed matter physics ${ }^{2}$ from the view of potential application of MBS to the topological quantum computation., To date, we have known several promising systems hosting MFs such as spin-triplet $p$-wave superconductors, ${ }^{5-8}$ topological insulator/superconductor heterostructures, ${ }^{9}$ semiconductor/superconductor junctions with strong spin-orbit coupling, ${ }^{10-13}$ helical superconductors, ${ }^{14}$ and superconducting topological insulators. ${ }^{15}$ The most attractive case among them is the semiconductor nanowire fabricated on top of a superconductor because of its easy tunability of MBS by changing the chemical potential in the nanowire and by applying the Zeeman field onto it. ${ }^{16,17}$ Actually, numerous theoretical studies have discussed MFs or MBSs in such nanowires. ${ }^{18,19}$ The zero-bias conductance peak reported very recently would be considered as an evidence of MFs (MBS). ${ }^{16,17,20}$ These researches have stimulated a number of theoretical investigations on unusual charge transport phenomena through the MBS in normal-metal/superconductor (NS) and superconductor/normal-metal/superconductor (SNS) junctions on nanowires. ${ }^{21}$ However, no studies have ever tried to analyze features of Cooper pairs which support the anomalous transport properties. We address this issue in this paper.

Odd-frequency Cooper pairing was originally proposed to understand the nature of unconventional superfluidity and superconductivity. ${ }^{22}$ The ubiquitous appearance of the odd-frequency pairs at the surface of superconductors and near the interface of superconducting junctions has been established and widely accepted in recent years. ${ }^{23}$ The zero-energy Andreev bound state (ABS) at the surface of unconventional superconductors ${ }^{25}$ is reinterpreted in terms of the odd-frequency Cooper pairing. ${ }^{24}$ In particular, the oddfrequency Cooper pairs make the background of the anomalous proximity effect in a diffusive normal metal attached to a spintriplet superconductor: ${ }^{26}$ (i) the large zero-energy quasiparticle density of states in a normal metal, ${ }^{27,28}$ (ii) the quantized zero-bias conductance at twice Sharvin's value in diffusive NS junctions, ${ }^{28}$ (iii) the fractional current $(J)$-phase $(\varphi)$ relationship of $J \propto \sin (\varphi / 2)$ in diffusive SNS junctions, ${ }^{29}$ (iv) the zero-bias anomaly in nonlocal conductance spectra, ${ }^{30}$ and (v) the anomalous surface impedance in NS bilayers. ${ }^{31}$

In this paper, we show that disordered NS and SNS junctions of nanowire indicate the properties of (i)-(iii) when the superconducting nanowire is topologically nontrivial. In addition, the amplitude of odd-frequency pairs in the normal nanowire suddenly grows as soon as the superconducting nanowire undergoes the transition to topologically nontrivial phase. The unusual transport phenomena due to the MFs (Ref. 20) are nothing but the anomalous proximity effect due to the odd-frequency pairs. We will conclude that the odd-frequency Cooper pairs are indispensable to realizing MFs in solids.

\section{TRANSPORT PROPERTIES}

Let us consider a nanowire with strong spin-orbit coupling fabricated on a junction of an insulator and a metallic superconductor as shown in Fig. 1. A segment on the insulator and that on the superconductor are in the normal and the superconducting states, respectively. The diameter of the nanowire is sufficiently small so that the number of propagating channels is unity for each spin degree of freedom. We describe the present nanowire by using the tight-binding model in one-dimension, for noninteracting electrons, ${ }^{32}$

$$
\begin{gathered}
H_{0}=-t \sum_{j, \alpha}\left(c_{j+1, \alpha}^{\dagger} c_{j, \alpha}+c_{j, \alpha}^{\dagger} c_{j+1, \alpha}\right) \\
+i \frac{\lambda}{2} \sum_{j, \alpha, \beta}\left[c_{j+1, \alpha}^{\dagger}\left(\hat{\sigma}_{2}\right)_{\alpha, \beta} c_{j, \beta}-c_{j, \alpha}^{\dagger}\left(\hat{\sigma}_{2}\right)_{\alpha, \beta} c_{j+1, \beta}\right] \\
+\sum_{j, \alpha, \beta} c_{j, \alpha}^{\dagger}\left\{(2 t-\mu) \hat{\sigma}_{0}-V_{e x} \hat{\sigma}_{3}\right\}_{\alpha, \beta} c_{j, \beta}, \\
H_{s}=\sum_{j \geqslant L+1}\left[\Delta e^{i \varphi} c_{j, \uparrow}^{\dagger} c_{j, \downarrow}^{\dagger}+\text { H.c. }\right] \\
H_{d}=\sum_{1 \leqslant j \leqslant L, \alpha} V_{j} c_{j, \alpha}^{\dagger} c_{j, \alpha}, \\
H_{s 2}=\sum_{j \leqslant 0}\left[\Delta e^{i \varphi_{2}} c_{j, \uparrow}^{\dagger} c_{j, \downarrow}^{\dagger}+\text { H.c. }\right],
\end{gathered}
$$




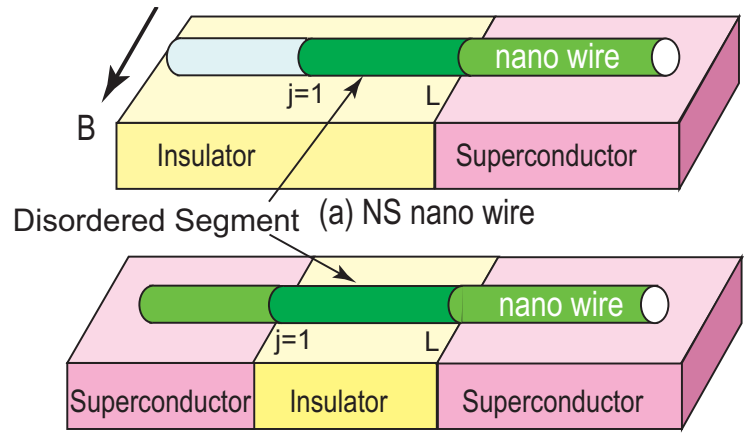

(b) SNS nano wire

FIG. 1. (Color online) Schematic pictures of NS and SNS junctions.

where $c_{j, \alpha}^{\dagger}\left(c_{j, \alpha}\right)$ is the creation (annihilation) operator of an electron at the lattice site $j$ with $\operatorname{spin} \alpha=(\uparrow$ or $\downarrow), t$ denotes the hopping integral, $\mu$ is the chemical potential, and $\Delta$ is the pair potential in the superconducting segment. The Pauli matrices in spin space are denoted by $\hat{\sigma}_{j}$ for $j=1-3$ and the unit matrix of $2 \times 2$ is $\hat{\sigma}_{0}$. The onsite potential in the normal segment is given randomly in the range of $-W / 2 \leqslant V_{j} \leqslant W / 2$. We measure the energy and the length in units of $t$ and the lattice constant, respectively. Throughout this paper, we fix several parameters as $\mu=t, W=2 t$, and the pair potential at the zero temperature $\Delta=0.01 t$. The number of samples used for the random ensemble averaging is typically $10^{3}-10^{5}$. By tuning the magnetic field $B$ as shown in Fig. 1, it is possible to introduce external Zeeman potential $V_{e x}$. For $V_{e x}>V_{c} \equiv$ $\sqrt{\Delta_{0}^{2}+\mu^{2}}$, the number of propagating channels becomes unity and the superconducting segment undergoes the transition to topologically nontrivial phase. In the tight-binding model, the finite bandwidth gives an additional condition $V_{e x}<V_{c 2} \equiv$ $4 t-\mu$ for the topological phase. Here, we briefly summarize calculated results of the normal conductance of the disordered nanowires with using the recursive Green's function method. ${ }^{33}$ By analyzing the Hamiltonian $H_{0}+H_{d}$, we confirmed that the normal conductance decays exponentially with increasing $L .{ }^{34}$ This is because one-dimensional disordered wires are basically in the localization regime.

At first, we calculate the differential conductance $G_{\mathrm{NS}}$ of NS junctions based on the standard formula ${ }^{35}$

$$
G_{\mathrm{NS}}=\frac{e^{2}}{h} \sum_{\alpha, \beta}\left[\delta_{\alpha, \beta}-\left|r_{\alpha, \beta}^{e e}\right|^{2}+\left|r_{\alpha, \beta}^{h e}\right|^{2}\right]_{E=e V},
$$

where we consider the Hamiltonian $H_{0}+H_{d}+H_{s}$. In Eq. (5), $r_{\alpha, \beta}^{e e}$ and $r_{\alpha, \beta}^{h e}$ are the normal and Andreev reflection coefficients of the junction at energy $E$ measured from the Fermi level. We show $G_{\mathrm{NS}}$ in units of $G_{Q}=2 e^{2} / h$ as a function of the bias voltage $e V$ for the nontopological and the topological nanowires in Figs. 2(a) and 2(b), respectively. The length of disordered segment $L$ is chosen as 10, 20, and 50 lattice constants. The conductance for the nontopological nanowires ( $V_{e x}=\lambda=0$ ) in Fig. 2(a) decreases with increasing the length of disordered segment $L$ for all $e V$. The similar tendency can be seen also in the conductance of the topological nanowires ( $V_{e x}=1.5 t$ and $\lambda=0.5 t$ ) in Fig. 2(b) for finite $e V$. However, the zero-bias conductance of the topological nanowires is
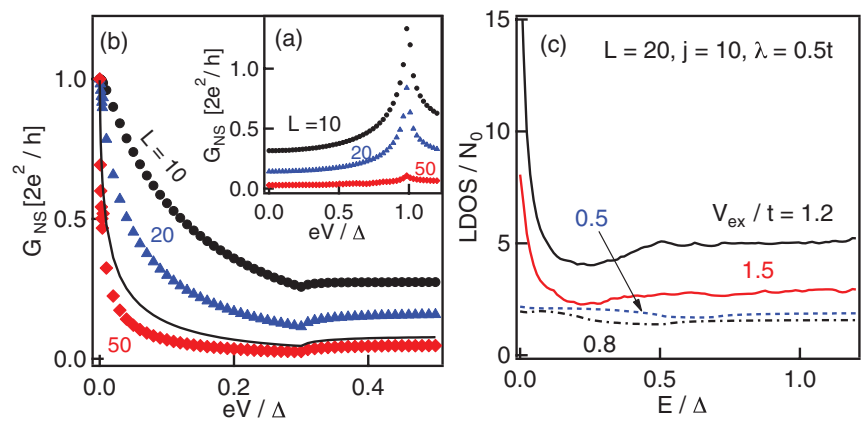

FIG. 2. (Color online) The differential conductance of NS nanowires is plotted as a function of the bias voltage for nontopological nanowire $\left(V_{e x}=\lambda=0\right)$ in (a) and for the topological nanowire ( $V_{e x}=1.5 t$ and $\lambda=0.5 t$ ) in (b). In (b), we also plot the results for $W / t=4$ and $L=10$ with a solid line. The local density of states at the center of the disordered segment $(j=10)$ are shown for several $V_{e x}$ in (c) with $\lambda=0.5 t$, where we add a small imaginary part $i \delta_{\epsilon}$ with $\delta_{\epsilon}=0.01 \Delta$ to the energy.

quantized at $G_{Q}$ irrespective of $L$, which is an intrinsic phenomenon in the presence of MF. The results suggest a perfect transmission channel due to the penetration of a resonant state into the disordered segment. The local density of states (LDOS) in the disordered nanowire supports this statement as shown in Fig. 2(c), where we plot the LDOS at the center of the disordered segment $(j=10)$ as a function of $E$ for $L=20$ and $\lambda=0.5 t$. The results are normalized to the density of states at the Fermi level in clean normal nanowire $N_{0}$. The LDOS for $V_{e x}>V_{c}$ show the large zero-energy peak reflecting the MBS as shown in the results for $V_{e x} / t=1.5$ and 1.2. On the other hand, the LDOS for $V_{e x} / t=0.5$ and 0.8 are almost flat around the zero energy.

Second, we explain why the superconducting nanowire shows the anomalous proximity effect which is unique to the $p_{x}$-wave spin-triplet superconductor. The singleparticle Hamiltonian in Eq. (1) is essentially equivalent to $\hat{h}_{0}(k)=\xi_{k} \hat{\sigma}_{0}-\lambda k \hat{\sigma}_{2}-V_{e x} \hat{\sigma}_{3}$ in momentum space with $\xi_{k}=$ $\hbar^{2} k^{2} /(2 m)-\mu$. By applying a unitary transformation diagonalizing $h_{0}(k)$ and $-h_{0}^{*}(-k)$, Bogoliubov-de Gennes (BdG) Hamiltonian of the nanowire reduces to $2 \times 2$ Hamiltonian for $V_{e x}>V_{c}$ (see also Appendix A for details):

$$
\left[\begin{array}{cc}
\hat{h}_{0}(k) & i \Delta \hat{\sigma}_{2} \\
-i \Delta^{*} \hat{\sigma}_{2} & -\hat{h}_{0}^{*}(-k)
\end{array}\right] \rightarrow\left[\begin{array}{cc}
\xi_{k}-A & \tilde{\Delta}_{k} e^{i \pi / 2} \\
\tilde{\Delta}_{k} e^{-i \pi / 2} & -\xi_{k}+A
\end{array}\right],
$$

with $A=\sqrt{V_{e x}^{2}+(\lambda k)^{2}}$ and $\tilde{\Delta}_{k}=\Delta \lambda k / A$ because a spin branch pinches off from the Fermi level (i.e., $\xi+A>0$ ). The right-hand side of Eq. (6) is equivalent to the $\mathrm{BdG}$ Hamiltonian of spinless $p_{x}$-wave superconductor in one dimension when we focus on low-energy excitation after redefining the chemical potential $\mu+A \rightarrow \mu$ and the pair potential $\tilde{\Delta}_{k} e^{i \pi / 2} \rightarrow \Delta\left(k / k_{F}\right)$ with $k_{F}$ being the Fermi wave number. Therefore, the physics in the topological nanowire is the same as that of $p_{x}$-wave superconductor. In fact, we have confirmed that the Josephson current in SNS junctions of disordered nanowire shows the fractional current-phase relationship at low temperature as shown in Fig. 3(a). ${ }^{29}$ Here, we attach the second supercondutor for $j \leqslant 0$ by adding $H_{s 2}$ to $H_{0}+H_{s}+H_{d}$, and plot the Josephson current $J$ as a 

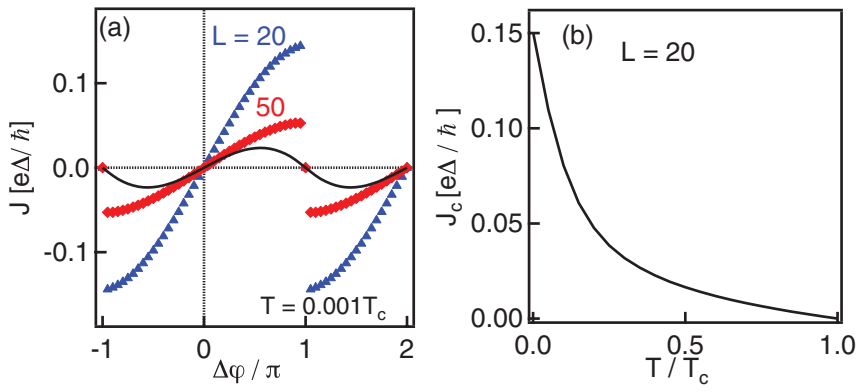

FIG. 3. (Color online) (a) Current-phase relationship in SNS junctions of topological wire at $T / T_{c}=0.001$ for $V_{e x}=1.5 t$ and $\lambda=0.5 t$. For comparison, the results for nontopological wire $\left(V_{e x}=\right.$ $\lambda=0$ and $L=50$ ) is plotted with a solid line. (b) The Josephson critical current versus temperature in a topological nanowire.

function of $\Delta \varphi=\varphi_{2}-\varphi$ at $T=0.001 T_{c}$ for $V_{e x}=1.5 t$ and $\lambda=0.5 t$. The results show $J \propto \sin (\Delta \varphi / 2)$ for $-\pi \leqslant \Delta \varphi \leqslant \pi$ irrespective of $L$. For comparison, we also plot the results for $V_{e x}=\lambda=0$ and $L=50$ with a solid line which shows usual sinusoidal current-phase relationship. Correspondingly, the Josephson critical current plotted as a function of temperature shows the so-called low-temperature anomaly ${ }^{29}$ in Fig. 3(b).

\section{ODD-FREQUENCY PAIRS}

Next, we discuss the relationship between Majorana fermions and odd-frequency Cooper pairs by analyzing the Green's functions in junctions of the $p_{x}$-wave supercondutor. A semi-infinite wire of the $p_{x}$-wave superconductor occupying $x>0$ hosts a Majorana fermion around its edge at $E=0$. Solving the $\mathrm{BdG}$ equation, the wave function of such surface state is calculated to be $\phi_{0}(x)^{T}=\left[u_{0}(x), v_{0}(x)\right]^{T}$, where $u_{0}(x)=C(x) \chi, v_{0}(x)=$ $C(x) \chi^{*}, C(x)=\sqrt{2 / \xi_{0}} e^{-x / 2 \xi_{0}} \sin (k x), \chi=e^{i \pi / 4} e^{i \varphi / 2}$, and $\xi_{0}$ is the coherence length. Details are shown in Appendix B. The electron operator includes the contribution from such surface state $\psi_{0}(x)$ as represented by $\psi_{0}(x)=\chi \gamma(x), \psi_{0}^{\dagger}(x)=$ $\chi^{*} \gamma(x)$ with $\gamma(x)=C(x)\left(\gamma_{0}+\gamma_{0}^{\dagger}\right)$. Here, $\gamma_{0}$ is the annihilation operator of the Majorana bound state. The special relation $v_{0}(x)=u_{0}^{*}(x)$ plays a crucial role in the Majorana relation of $\gamma(x)=\gamma^{\dagger}(x){ }^{24}$ As a result, the two Green's functions calculated for $|E| \ll \Delta$,

$$
\begin{aligned}
& g\left(E ; x, x^{\prime}\right) \approx \frac{u_{0}(x) u_{0}^{*}\left(x^{\prime}\right)+v_{0}^{*}(x) v_{0}\left(x^{\prime}\right)}{E+i \delta_{\epsilon}}, \\
& f\left(E ; x, x^{\prime}\right) \approx \frac{u_{0}(x) v_{0}^{*}\left(x^{\prime}\right)+v_{0}^{*}(x) u_{0}\left(x^{\prime}\right)}{E+i \delta_{\epsilon}},
\end{aligned}
$$

depend on each other. Since $v_{0}(x)=u_{0}^{*}(x)$, they satisfy

$$
g\left(E, x, x^{\prime}\right)=\left(\chi^{*}\right)^{2} f\left(E, x, x^{\prime}\right)=I\left(E ; x, x^{\prime}\right) .
$$

This relation directly bridges Majorana fermions and oddfrequency Cooper pairs. The real (imaginary) part of $\left(\chi^{*}\right)^{2} f(E ; x, x)$ is an odd (even) function of $E$, which represents the odd-frequency symmetry of Cooper pairs. The orbital part is $s$-wave symmetry when $f$ is calculated at $x=x^{\prime}$. In fact, the imaginary part of $g(E ; x, x)$ must be even function of $E$ because it represents LDOS of the Majorana bound state. It is possible to check this argument in a junction which consists

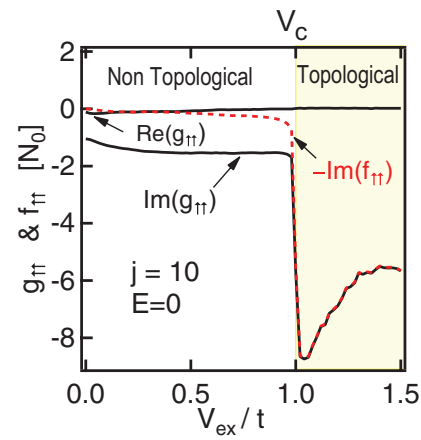

FIG. 4. (Color online) The normal $g_{\uparrow}$ and the anomalous $f_{\uparrow}$ Green's function for $E=0$ at $j=10$, with $L=20$. We introduce a small imaginary part $\delta_{\epsilon}=0.001 \Delta$.

of a normal metal $(x<0)$ and a $p_{x}$-wave superconductor $(x>0)$ in one dimension ${ }^{36}$ as shown in Appendix B. At the NS interface $(x=0)$, we introduce a potential barrier $V_{0} \delta(x)$ whose normal transmission coefficient is $t_{n}=k_{F} /\left(k_{F}+i z_{0}\right)$ with $z_{0}=V_{0} / \hbar v_{F}$. When we focus on the subgap energy $|E| \ll \Delta$ in the tunneling limit $\left|t_{n}\right| \ll 1$, we find that the Green's functions in the superconductor $x>0$ satisfy Eq. (9) and become

$$
I(E ; x, x) \approx \frac{\pi N_{0} \Delta}{E+i \Delta\left|t_{n}\right|^{2} / 2} e^{-x / \xi_{0}} \sin ^{2}(k x) .
$$

For comparison, we show the anomalous Green's function in a uniform $p_{x}$-wave superconductor:

$$
\left(\chi^{*}\right)^{2} f\left(E ; x, x^{\prime}\right)=-i \frac{\pi N_{0}}{2} \frac{\Delta \sin k\left(x-x^{\prime}\right)}{\sqrt{\left(E+i 0^{+}\right)^{2}-\Delta^{2}}},
$$

with $\sqrt{\left(E+i 0^{+}\right)^{2}-\Delta^{2}}$ being $\operatorname{sgn}(E) \sqrt{E^{2}-\Delta^{2}}$ for $|E|>\Delta$ and $i \sqrt{\Delta^{2}-E^{2}}$ for $|E|<\Delta$, where we assume $\left|x-x^{\prime}\right| \ll$ $\xi_{0}$. The anomalous Green's function satisfies $f\left(x-x^{\prime}\right)=$ $-f\left(x^{\prime}-x\right)$ reflecting the odd-parity symmetry. In contrast to Eq. (10), the real (imaginary) part of $\left(\chi^{*}\right)^{2} f\left(E, x, x^{\prime}\right)$ is an even (odd) function of $E$, which represents the even-frequency symmetry.

The important relation in Eq. (9) can be confirmed in the normal segment of NS nanowire as shown in Fig. 4, where we fix the energy at $E=0$ and plot $g_{\uparrow}(j, j)$ and $-f_{\uparrow \uparrow}(j, j)$ at the center of the normal segment $j=10$. We note that an extra phase factor $\varphi=\pi / 2$ in Eq. (6) makes $\left(\chi^{*}\right)^{2}=-1$ in Eq. (9). For $V_{e x}>V_{c}$, the results show $\operatorname{Im}\left(g_{\uparrow \uparrow}\right)=-\operatorname{Im}\left(f_{\uparrow}\right)$. The real part of $f_{\uparrow \uparrow}$ is always zero at $E=0$ due to the odd-frequency symmetry. Correspondingly, $\operatorname{Re}\left(g_{\uparrow}\right)$ also goes to zero for $V_{e x}>V_{c}$. In addition, $-\operatorname{Im}\left(g_{\uparrow \uparrow}\right)=\operatorname{Im}\left(f_{\uparrow \uparrow}\right)$ suddenly increases as $V_{e x}$ increasing across $V_{c}$, which corresponds to the zeroenergy peak in LDOS in Fig. 2(c). The results demonstrate the penetration of Majorana fermions and odd-frequency Cooper pairs into the normal disordered segment at the same time.

In the NS junction of $p_{x}$-wave superconductor, it is possible to calculate exactly the wave function in the presence of a single impurity $V_{i} \delta\left(x-x_{i}\right)$ in the normal metal by solving the Lippmann-Schwinger equation ${ }^{36}$ as shown in Appendix B:

$$
\phi_{n}(x)=\phi_{n}^{i n i}(x)+\hat{G}\left(E ; x, x_{i}\right) V_{i} \hat{\sigma}_{3} \phi_{n}\left(x_{i}\right),
$$

where $\phi_{n}(x)$ is the wave function in the presence of the impurity and $\left[\phi_{n}^{i n i}(x)\right]^{T}=\left[e^{i k x}+r_{e e} e^{-i k x}, r_{h e} e^{i k x}\right]^{T}$ is that in 
the ballistic case with $r_{e e}$ and $r_{h e}$ being the normal and the Andreev reflection coefficients at the NS interface from the electron branch, respectively. By putting $x=x_{i}$, the equation in Eq. (12) has the closed form for $\phi_{n}\left(x_{i}\right)$, which results in

$$
\phi_{n}(x)=\phi_{n}^{i n i}(x)+\frac{1}{Y}\left[\begin{array}{c}
-e^{i k x} e^{2 i k x_{i}} z_{i}\left(z_{i}+i\right) X \\
e^{i k x}(1-Y) r_{h e}
\end{array}\right] \text {, }
$$

at $E=0$ for $x<x_{i}$, where $Y=1+z_{i}^{2} X, X=1-r_{h e} r_{e h}$, $z_{i}=V_{i} / \hbar v_{F}$, and $r_{e h}$ is the Andreev reflection coefficient of the NS interface from the hole branch. We have already taken into account the absence of the normal reflection at the NS interface at $E=0$ (i.e., $r_{e e}=r_{h h}=0$ ). At $E=0$, the Andreev reflection coefficients $r_{h e}=-i e^{-i \varphi}$ and $r_{e h}=i e^{i \varphi}$ satisfy an important relation $r_{e h} r_{h e}=1$ which eliminates the second term of Eq. (13). Thus, the zero-bias conductance quantization at $G_{Q}=2 e^{2} / h$ holds even in the presence of an impurity. The relation of $r_{e h} r_{h e}=1$ is nothing but the condition for forming the MBS at $E=0$. To discuss whole effects of scatterings by many impurities, we need to solve the nonlinear quasiclassical Usadel equation. ${ }^{27,28}$ The analytical expression of the zero-bias conductance also show $G_{\mathrm{NS}}=G_{Q}$ as shown in Appendix C. The diffusive normal metal is assumed in the Usadel equation. The validity of $G_{\mathrm{NS}}=G_{Q}$ in the localization regime is confirmed in numerical calculation in Figs. 2(a) and 6.

\section{CONCLUSION}

In summary, we have theoretically discussed the anomalous transport phenomena in NS and SNS junctions of nanowires in which the superconducting segment is topologically nontrivial and the normal segment is disordered by random impurity potential. The physics behind the anomalous transport can be understood in terms of the odd-frequency Cooper pairing. We conclude that Majorana fermions and odd-frequency Cooper pairs in solids are two sides of a same coin.

\section{ACKNOWLEDGMENT}

This work was supported by KAKENHI on Innovative Areas "Topological Quantum Phenomena" and KAKENHI (22540355, 22103005) from MEXT of Japan.

\section{APPENDIX A: TRANSFORMATION OF HAMILTONIAN}

The starting Hamiltonian of this paper is equivalent to

$$
\begin{gathered}
H_{N W}=\left[\begin{array}{cc}
\hat{h}_{k} & i \Delta \hat{\sigma}_{2} e^{i \varphi} \\
-i \Delta \hat{\sigma}_{2} e^{-i \varphi} & -\hat{h}_{-k}^{*}
\end{array}\right], \\
h_{k}=\xi_{k} \hat{\sigma}_{0}-V_{e x} \hat{\sigma}_{3}-\lambda k \hat{\sigma}_{2}, \quad \xi_{k}=\frac{\hbar^{2} k^{2}}{2 m}-\mu,
\end{gathered}
$$

where $\mu$ is the chemical potential, $V_{e x}$ is the Zeeman potential due to external magnetic field, $\lambda k \hat{\sigma}_{2}$ represents the spin-orbit coupling, $\hat{\sigma}_{0}$ is the unit matrix in spin space, and $\hat{\sigma}_{j}$ for $j=1-3$ are the Pauli's matrices.
By applying the following unitary transformation, ${ }^{37}$ the Hamiltonian is deformed as

$$
\begin{aligned}
& \check{D}^{\dagger} H_{N W} \check{D} \\
& =\left[\begin{array}{cccc}
\xi_{k}-A & 0 & \Delta_{\lambda} e^{i(\varphi+\pi / 2)} & \Delta_{V} e^{i \varphi} \\
0 & \xi_{k}+A & -\Delta_{V} e^{i \varphi} & \Delta_{\lambda} e^{i(\varphi-\pi / 2)} \\
\Delta_{\lambda} e^{-i(\varphi+\pi / 2)} & -\Delta_{V} e^{-i \varphi} & -\xi_{k}+A & 0 \\
\Delta_{V} e^{-i \varphi} & \Delta_{\lambda} e^{-i(\varphi-\pi / 2)} & 0 & -\xi_{k}-A
\end{array}\right], \\
& \Delta_{\lambda}=\Delta \frac{\lambda k}{A}, \quad \Delta_{V}=\Delta \frac{V_{e x}}{A}, \quad A=\sqrt{V_{e x}^{2}+(\lambda k)^{2}}, \\
& \check{D}=\left[\begin{array}{cc}
\hat{U} & 0 \\
0 & \hat{U}
\end{array}\right], \quad \hat{U}=\left[\begin{array}{cc}
\alpha & i \operatorname{sgn}(k) \beta \\
i \operatorname{sgn}(k) \beta & \alpha
\end{array}\right], \\
& \alpha=\sqrt{\frac{1}{2}\left(1+\frac{V_{e x}}{A}\right)}, \quad \beta=\sqrt{\frac{1}{2}\left(1-\frac{V_{e x}}{A}\right)} .
\end{aligned}
$$

The topologically nontrivial phase is characterized by $V_{e x}>$ $\sqrt{\mu^{2}+\Delta^{2}}$. In such case, only one dispersion remains at the Fermi level for each Nambu space (i.e., $\xi_{k}+A>0$ ). Therefore, the Hamiltonian reduces to $2 \times 2$ Nambu space as

$$
\hat{H}_{N W 2}=\left[\begin{array}{cc}
\xi_{k}-A & \Delta_{\lambda} e^{i(\varphi+\pi / 2)} \\
\Delta_{\lambda} e^{-i(\varphi+\pi / 2)} & -\xi_{k}+A
\end{array}\right] .
$$

When we focus on the low-energy quasiparticle excitation, this is equivalent to the Hamiltonian describing the equal spintriplet (spinless) $p_{x}$-wave superconductor

$$
\hat{H}_{p_{x}}=\left[\begin{array}{cc}
\xi_{k} & \Delta \frac{k}{k_{F}} e^{i \varphi} \\
\Delta \frac{k}{k_{F}} e^{-i \varphi} & -\xi_{k}
\end{array}\right] .
$$

Here, we redefine $\mu+A \rightarrow \mu$ in the diagonal term and $\Delta_{\lambda} e^{i \pi / 2} \rightarrow \Delta\left(k / k_{F}\right)$ in the off-diagonal term. In previous papers, ${ }^{26,27,29-31}$ we have studied the anomalous proximity effect starting from the Hamiltonian in Eq. (A8).

\section{APPENDIX B: ANALYSIS OF $p_{x}$-WAVE SUPERCONDUCTOR}

\section{Green's function and its representation}

The retarded Green's functions are defined by the standard way

$$
\begin{aligned}
& \hat{G}\left(x, t ; x^{\prime}, t^{\prime}\right) \\
& =-i \Theta\left(t-t^{\prime}\right)\left[\begin{array}{rr}
\left\{\psi(x, t), \psi^{\dagger}\left(x^{\prime} t^{\prime}\right)\right\} & \left\{\psi(x, t), \psi\left(x^{\prime} t^{\prime}\right)\right\} \\
\left\{\psi^{\dagger}(x, t), \psi^{\dagger}\left(x^{\prime} t^{\prime}\right)\right\} & \left\{\psi^{\dagger}(x, t), \psi\left(x^{\prime} t^{\prime}\right)\right\}
\end{array}\right] \\
& \quad=\left[\begin{array}{rr}
G\left(x, t ; x^{\prime} t^{\prime}\right) & F\left(x, t ; x^{\prime} t^{\prime}\right) \\
\tilde{F}\left(x, t ; x^{\prime} t^{\prime}\right) & \tilde{G}\left(x, t ; x^{\prime} t^{\prime}\right)
\end{array}\right]
\end{aligned}
$$

where $\psi(x)\left[\psi^{\dagger}(x)\right]$ is the annihilation (creation) operator of a spinless electron. In the case of spin-triplet superconductors, the electron operators are represented by the Bogoliubov 
transformation

$$
\left[\begin{array}{c}
\psi(x) \\
\psi^{\dagger}(x)
\end{array}\right]=\sum_{v}\left[\begin{array}{ll}
u_{v}(x) & v_{v}^{*}(x) \\
v_{v}(x) & u_{v}^{*}(x)
\end{array}\right]\left[\begin{array}{c}
\gamma_{v} \\
\gamma_{-v}^{\dagger}
\end{array}\right]
$$

where $\gamma_{\nu}$ is the annihilation operator of the Bogoliubov quasiparticle belonging to $E_{\nu}$. The wave functions $u_{v}(x)$ and $v_{\nu}(x)$ are obtained by solving the Bogoliubov-de Gennes $(\mathrm{BdG})$ equation. The Green's functions are expressed in spectral representation as

$$
\begin{aligned}
& G\left(E ; x, x^{\prime}\right)=\sum_{v}\left[\frac{u_{v}(x) u_{v}^{*}\left(x^{\prime}\right)}{E+i \delta-E_{v}}+\frac{v_{v}^{*}(x) v_{v}\left(x^{\prime}\right)}{E+i \delta+E_{v}}\right], \\
& F\left(E ; x, x^{\prime}\right)=\sum_{v}\left[\frac{u_{v}(x) v_{v}^{*}\left(x^{\prime}\right)}{E+i \delta-E_{v}}+\frac{v_{v}^{*}(x) u_{v}\left(x^{\prime}\right)}{E+i \delta+E_{v}}\right],
\end{aligned}
$$

where $i \delta$ is a small imaginary part.

\section{Uniform superconductor}

The retarded Green's function of a uniform spinless $p_{x}$ wave superconductor is calculated to be

$$
\begin{aligned}
& \hat{G}\left(E ; x, x^{\prime}\right)= \frac{-i \pi N_{0}}{2 \Omega} \hat{\Phi}\left[\left(\begin{array}{cc}
E+\Omega & \Delta s_{x} \\
\Delta s_{x} & E-\Omega
\end{array}\right) e^{i k^{+}\left|x-x^{\prime}\right|}\right. \\
&\left.+\left(\begin{array}{cc}
E-\Omega & -\Delta s_{x} \\
-\Delta s_{x} & E+\Omega
\end{array}\right) e^{-i k^{-}\left|x-x^{\prime}\right|}\right] \hat{\Phi}^{*}, \\
& \hat{G}\left(E ; x, x^{\prime}\right)=\left(\begin{array}{cc}
G\left(E ; x, x^{\prime}\right) & F\left(E ; x, x^{\prime}\right) \\
\tilde{F}\left(E ; x, x^{\prime}\right) & \tilde{G}\left(E ; x, x^{\prime}\right)
\end{array}\right) \\
& k^{ \pm}=k\left(1 \pm \frac{\Omega}{2 \mu}\right), \quad \Omega=\sqrt{(E+i \delta)^{2}-\Delta^{2}} \\
& \hat{\Phi}=\operatorname{diag}\left[e^{i \varphi / 2}, e^{-i \varphi / 2}\right], \quad s_{x}=\operatorname{sgn}\left(x-x^{\prime}\right)
\end{aligned}
$$

where $N_{0}$ is the density of states (DOS) at the Fermi level in the normal state. The Green's functions are calculated as

$$
\begin{aligned}
G\left(E ; x, x^{\prime}\right)= & -i \frac{\pi N_{0}}{2} e^{i k \Omega\left|x-x^{\prime}\right| /(2 \mu)} \\
& \times\left[\frac{E}{\Omega} \cos k\left(x-x^{\prime}\right)+i \sin k\left|x-x^{\prime}\right|\right], \\
-i e^{-i \varphi} F\left(E ; x, x^{\prime}\right) & \\
= & -i \frac{\pi N_{0}}{2} e^{i k \Omega\left|x-x^{\prime}\right| /(2 \mu)} \frac{\Delta}{\Omega} \sin k\left(x-x^{\prime}\right) .
\end{aligned}
$$

From the normal Green's functions, the local density of states (LDOS) is calculated to be

$$
N(E, x)=\frac{-1}{\pi} \operatorname{Im} \operatorname{Tr} \hat{G}(E, x, x)=N_{0} \operatorname{Re} \frac{E}{\Omega} .
$$

The LDOS is an even function of $E$ because of the relation

$$
\sqrt{(E+i \delta)^{2}-\Delta^{2}}= \begin{cases}\sqrt{E^{2}-\Delta^{2}}, & \Delta<E \\ i \sqrt{\Delta^{2}-E^{2}}, & 0<|E|<\Delta \\ -\sqrt{E^{2}-\Delta^{2}}, & E<-\Delta .\end{cases}
$$

It is evident that there is no subgap state in uniform superconductor. From the off-diagonal part, it is possible to check the pairing symmetry. The anomalous Green's function satisfies $F\left(x-x^{\prime}\right)=-F\left(x^{\prime}-x\right)$, which indicates the oddparity symmetry. In addition, the real part of $-i e^{-i \varphi} F\left(E, x, x^{\prime}\right)$ is an even function of $E$, whereas the imaginary part of it is an odd function of $E$. This means that Cooper pairs have the even-frequency symmetry.

\section{Majorana surface bound state}

Next, we consider a semi-infinite $p_{x}$-wave superconductor which occupies $x>0$ as shown in Fig. 1(a). By solving the Bogoliubov-de Gennes equation, the wave function for the subgap state is expressed by

$$
\begin{aligned}
\Psi_{S}(x)= & A\left[\begin{array}{c}
E+i \tilde{\Omega} \\
\Delta e^{-i \varphi}
\end{array}\right] e^{i k x} e^{-x / 2 \xi_{0}} \\
& +B\left[\begin{array}{c}
E-i \tilde{\Omega} \\
-\Delta e^{-i \varphi}
\end{array}\right] e^{-i k x} e^{-x / 2 \xi_{0}},
\end{aligned}
$$

where $\tilde{\Omega}=\sqrt{\Delta^{2}-E^{2}}, A$ and $B$ are constant. From the boundary condition at $x=0$ [i.e., $\Psi_{S}(x=0)=0$ ], we find that a subgap state exists at $E=0$ and that the wave function of it becomes

$$
\begin{gathered}
\Psi_{S}(x)=\left[\begin{array}{l}
u_{0}(x) \\
v_{0}(x)
\end{array}\right]=C(x)\left[\begin{array}{c}
e^{i \pi / 4} e^{i \varphi / 2} \\
e^{-i \pi / 4} e^{-i \varphi / 2}
\end{array}\right], \\
C(x)=\sqrt{\frac{2}{\xi_{0}}} e^{-x / 2 \xi_{0}} \sin (k x) .
\end{gathered}
$$

We note that two components in the wave function satisfy an important relation

$$
u_{0}(x)=v_{0}^{*}(x)
$$

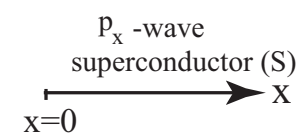

(a)

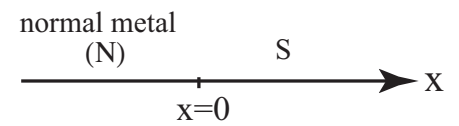

(b)

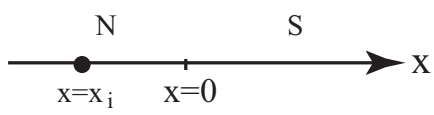

(c)

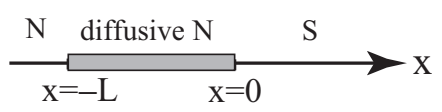

(d)

FIG. 5. System under consideration. (a) A semi-infinite $p_{x}$ wave superconductor. (b) A clean normal-metal/superconductor (NS) junction of $p_{x}$-wave symmetry. (c) An impurity is introduced in the normal metal. (d) A diffusive normal metal is introduced in the NS junction. 
The BdG transformation reads as

$$
\begin{gathered}
{\left[\begin{array}{c}
\psi(x) \\
\psi^{\dagger}(x)
\end{array}\right]=\sum_{v \neq 0}\left[\begin{array}{ll}
u_{v}(x) & v_{v}^{*}(x) \\
v_{v}(x) & u_{v}^{*}(x)
\end{array}\right]\left[\begin{array}{c}
\gamma_{v} \\
\gamma_{-v}^{\dagger}
\end{array}\right]+\left[\begin{array}{c}
\phi_{0}(x) \\
\phi_{0}^{\dagger}(x)
\end{array}\right],} \\
{\left[\begin{array}{l}
\phi_{0}(x) \\
\phi_{0}^{\dagger}(x)
\end{array}\right]=\left[\begin{array}{ll}
u_{0}(x) & v_{0}^{*}(x) \\
v_{0}(x) & u_{0}^{*}(x)
\end{array}\right]\left[\begin{array}{c}
\gamma_{0} \\
\gamma_{0}^{\dagger}
\end{array}\right],}
\end{gathered}
$$

where $\gamma_{0}$ is the annihilation operator of the bound state. Together with Eqs. (B16) and (B17), we find

$$
\begin{gathered}
\phi_{0}(x)=e^{i \pi / 4} e^{i \varphi / 2} \gamma(x), \\
\phi_{0}^{\dagger}(x)=e^{-i \pi / 4} e^{-i \varphi / 2} \gamma(x), \\
\gamma(x)=C(x)\left(\gamma_{0}+\gamma_{0}^{\dagger}\right) .
\end{gathered}
$$

The fermion operator $\gamma(x)$ satisfies the Majorana relation $\gamma(x)=\gamma^{\dagger}(x)$. When we focus on $|E| \ll \Delta$, the contributions from $v=0$ become dominant in Eqs. (B4) and (B5). Near $E=0$, the normal and the anomalous Green's functions satisfy a relation

$$
G\left(E ; x, x^{\prime}\right)=-i e^{-i \varphi} F\left(E ; x, x^{\prime}\right)
$$

because they are calculated from Eq. (B19) as

$$
\begin{gathered}
G\left(E ; x, x^{\prime}\right)=\frac{2 C(x) C\left(x^{\prime}\right)}{E+i \delta}, \\
F\left(E ; x, x^{\prime}\right)=\frac{2 C(x) C\left(x^{\prime}\right)}{E+i \delta} i e^{i \varphi} .
\end{gathered}
$$

Equation (B23) directly relates Majorana fermions and oddfrequency Cooper pairs. When we consider $x=x^{\prime}, F(E, x, x)$ represents the pairing function of $s$-wave symmetry. The real part of $-i e^{-i \varphi} F(E ; x, x)$ is an odd function of $E$ and the imaginary part of it is an even function of $E$. This indicates that Cooper pairs have the odd-frequency symmetry. It is possible to confirm Eq. (B23) in NS junctions as discussed below.

\section{NS junction of $p_{x}$ superconductor}

It is possible to calculate the Green's function of a junction which consists of a normal metal $(x<0)$ and a $p_{x}$-wave superconductor $(x>0)$ in one dimension as shown in Fig. 5(b). In the superconductor, the retarded Green's function becomes $^{36}$

$$
\begin{aligned}
& \hat{G}_{s s}\left(E ; x, x^{\prime}\right)=\hat{\Phi} i \frac{N_{0} \pi}{2} \frac{E}{\Omega}\left[\left(\begin{array}{cc}
u^{2} & u v s_{x} \\
u v s_{x} & v^{2}
\end{array}\right) e^{i k^{+}\left|x-x^{\prime}\right|}+\left(\begin{array}{cc}
v^{2} & -u v s_{x} \\
-u v s_{x} & u^{2}
\end{array}\right) e^{-i k^{-}\left|x-x^{\prime}\right|}+\left(\begin{array}{cc}
-u v & v^{2} \\
u^{2} & -u v
\end{array}\right) e^{-i k^{-} x+i k^{+} x^{\prime}} r_{s s}^{h e}\right. \\
& \left.+\left(\begin{array}{cc}
u v & u^{2} \\
v^{2} & u v
\end{array}\right) e^{\left.i k^{+} x-i k^{-} x^{\prime}\right)} r_{s s}^{e h}+\left(\begin{array}{cc}
u^{2} & -u v \\
u v & -v^{2}
\end{array}\right) e^{i k^{+}\left(x+x^{\prime}\right)} r_{s s}^{e e}+\left(\begin{array}{cc}
-v^{2} & -u v \\
u v & u^{2}
\end{array}\right) e^{-i k^{-}\left(x+x^{\prime}\right)} r_{s s}^{h h}\right] \hat{\Phi}^{*}, \\
& \hat{G}_{s s}\left(E ; x, x^{\prime}\right)=\left(\begin{array}{cc}
G_{s s}\left(E ; x, x^{\prime}\right) & F_{s s}\left(E ; x, x^{\prime}\right) \\
\tilde{F}_{s s}\left(E ; x, x^{\prime}\right) & \tilde{G}_{s s}\left(E ; x, x^{\prime}\right)
\end{array}\right), \quad \hat{\Phi}=\operatorname{diag}\left(e^{i \varphi / 2}, e^{-i \varphi / 2}\right), \\
& u(v)=\sqrt{\frac{1}{2}\left(1+(-) \frac{\Omega}{E}\right)}, \quad \Omega=\sqrt{(E+i \delta)^{2}-\Delta^{2}}, \quad k^{ \pm}=k\left(1 \pm \frac{\Omega}{2 \mu}\right), \quad s_{x}=\operatorname{sgn}\left(x-x^{\prime}\right)
\end{aligned}
$$

for $x, x^{\prime}>0$. The subscript $s s$ in the Green's function $\hat{G}_{s s}\left(E ; x, x^{\prime}\right)$ means that both $x>0$ and $x^{\prime}>0$ indicate places in the superconductor. The normal and Andreev reflection coefficients are given by

$$
\begin{gathered}
r_{s s}^{h e}=\frac{u v}{\Xi}\left(2-\left|t_{n}\right|^{2}\right)=-r_{s s}^{e h}, \quad r_{s s}^{e e}=\frac{r}{\Xi}\left(u^{2}-v^{2}\right), \\
r_{s s}^{h h}=\frac{r^{*}}{\Xi}\left(u^{2}-v^{2}\right), \quad \Xi=1-\left|t_{n}\right|^{2} v^{2}, \\
t_{n}=\frac{k}{k+i z_{0}}, \quad r_{n}=\frac{-i z_{0}}{k+i z_{0}}, \quad z_{0}=V_{0} / \hbar v_{F},
\end{gathered}
$$

where $t_{n}$ and $r_{n}$ are the normal transmission coefficients due to the potential barrier at the interface described by $V_{0} \delta(x)$. When we focus on the subgap energy in the tunneling limit (i.e., $|E| \ll \Delta$ and $\left|t_{n}\right| \ll 1$ ), we find

$$
\begin{aligned}
G_{s s}(E ; x, x) & =-i e^{-i \varphi} F_{s s}(E ; x, x) \\
& \approx \pi N_{0} \frac{\Delta}{E+i \Delta\left|t_{n}\right|^{2} / 2} e^{-x / \xi_{0}} \sin ^{2}(k x) .
\end{aligned}
$$

The imaginary part of $G_{s s}(E ; x, x)$ gives the local density of the Andreev bound state and must be an even function of $E$. Therefore, the real part of $-i e^{-i \varphi} F_{s s}(E ; x, x)$ is an odd function of $E$ and the imaginary part of it is an even function of $E$, which indicates the odd-frequency symmetry. The condition in Eq. (B17) leads to the Majorana relation in operators at Eq. (B21). Equation (B21) results in Eq. (B23). Then, Eq. (B23) guarantees the odd-frequency symmetry of Cooper pairs. The orbital part of Cooper pairs is $s$-wave symmetry because $-i e^{-i \varphi} F_{s s}$ is calculated at $x=x^{\prime}$. 


\section{Perfect transmission at $E=0$}

In the normal metal of NS junction $\left(x, x^{\prime}<0\right)$, the Green's function is given by ${ }^{36}$

$$
\begin{gathered}
\hat{G}_{n n}\left(E ; x, x^{\prime}\right)=-i \frac{\pi N_{0}}{2}\left[\begin{array}{cc}
e^{-i k\left|x-x^{\prime}\right|}+e^{-i k\left(x+x^{\prime}\right)} r_{n n}^{e e} & e^{-i k x+i k x^{\prime}} r_{n n}^{e h} \\
e^{i k x-i k x^{\prime}} r_{n n}^{h e} & e^{i k\left|x-x^{\prime}\right|}+e^{i k\left(x+x^{\prime}\right)} r_{n n}^{h h}
\end{array}\right], \\
r_{n n}^{h e}=\frac{\left|t_{n}\right|^{2} e^{-i \varphi} u v}{\Xi}, \quad r_{n n}^{e h}=-\frac{\left|t_{n}\right|^{2} e^{i \varphi} u v}{\Xi}, \quad r_{n n}^{e e}=\frac{r_{n}}{\Xi}, \quad r_{n n}^{h h}=\frac{r_{n}^{*}}{\Xi}, \\
\hat{G}_{n n}\left(E ; x, x^{\prime}\right)=\left(\begin{array}{cc}
G_{n n}\left(E ; x, x^{\prime}\right) & F_{n n}\left(E ; x, x^{\prime}\right) \\
\tilde{F}_{n n}\left(E ; x, x^{\prime}\right) & \tilde{G}_{n n}\left(E ; x, x^{\prime}\right)
\end{array}\right) .
\end{gathered}
$$

The subscript $n n$ in the Green's function $\hat{G}_{n n}\left(E ; x, x^{\prime}\right)$ means that both $x<0$ and $x^{\prime}<0$ indicate places in the normal metal. We also confirmed the relation between the Green's function in the normal metal

$$
\begin{aligned}
G_{n n}(E ; x, x) & =-i e^{-i \varphi} F_{n n}(E ; x, x) \\
& \approx \pi N_{0} \frac{\Delta\left|t_{n}\right|^{2}}{E+i \Delta\left|t_{n}\right|^{2} / 2}
\end{aligned}
$$

for $|E| \ll \Delta$ and $\left|t_{n}\right|^{2} \ll 1$. As we discussed in Eq. (B23), this suggests the presence of Majorana fermions and odd-frequency Cooper pairs in the normal metal.

It is possible to calculate exactly the wave function where a single impurity $V_{i} \delta\left(x-x_{i}\right)$ exists in the normal metal as shown in Fig. 5(c) by using the Lippmann-Schwinger equation

$$
\begin{gathered}
\phi_{n}(x)=\phi_{n}^{i n i}(x)+\hat{G}_{n n}\left(E ; x, x_{i}\right) V_{i} \hat{\sigma}_{3} \phi_{n}\left(x_{i}\right), \\
\phi_{n}^{i n i}(x)=\left[\begin{array}{c}
e^{i k x}+e^{-i k x} r_{n n}^{e e} \\
e^{i k x} r_{n n}^{h e}
\end{array}\right],
\end{gathered}
$$

where $\phi_{n}^{i n i}(x)$ is the wave function in the ballistic case and $\phi_{n}(x)$ is that in the presence of the impurity. By solving this equation at $x=x_{i}$, we obtain

$$
\phi_{n}\left(x_{i}\right)=\left[1-\hat{G}_{n n}\left(E ; x_{i}, x_{i}\right) V_{i} \hat{\sigma}_{3}\right]^{-1} \phi_{n}^{i n i}\left(x_{i}\right) .
$$

The wave function for $x<x_{i}$ in the presence of the single impurity is expressed by

$$
\begin{aligned}
\phi_{n}(x) & =\phi_{n}^{i n i}(x)+\hat{G}_{n n}\left(E ; x, x_{i}\right) V_{i} \hat{\sigma}_{3}\left[1-\hat{G}_{n n}\left(E ; x_{i}, x_{i}\right) V_{i} \hat{\sigma}_{3}\right]^{-1} \phi_{n}^{i n i}\left(x_{i}\right) \\
& =\phi_{n}^{i n i}(x)+\frac{1}{Y}\left[\begin{array}{c}
e^{-i k x}\left\{-i z_{i}\left(B_{1}^{2}-e^{2 i k x_{i}} r_{n n}^{h e} r_{n n}^{e h}\right)\right\}-z_{i}^{2}\left\{B_{1} B_{2}-r_{n n}^{h e} r_{n n}^{e h}\right\} e^{i k x_{i}} B_{1} \\
e^{i k x}(1-Y) r_{n n}^{h e}
\end{array}\right], \\
Y & =1+z_{i}\left(e^{i 2 k x_{i}} r_{n n}^{h h}-e^{-i 2 k x_{i}} r_{n n}^{e e}\right)+z_{i}^{2}\left(1-r_{n n}^{h e} r_{n n}^{e h}+e^{i 2 k x_{i}} r_{n n}^{h h}+e^{-i 2 k x_{i}} r_{n n}^{e e}\right), \\
B_{1} & =e^{i k x_{i}}+e^{-i k x_{i}} r_{n n}^{e e}, \quad B_{2}=e^{-i k x_{i}}+e^{i k x_{i}} r_{n n}^{h h}, \quad z_{i}=V_{i} / \hbar v
\end{aligned}
$$

At $E=0$, the reflection coefficients become

$$
r_{n n}^{e h}=i e^{i \varphi}, \quad r_{n n}^{h e}=-i e^{-i \varphi}, \quad r_{n n}^{e e}=r_{n n}^{h h}=0
$$

These relations immediately lead to

$$
B_{1}^{2}-e^{2 i k x_{i}} r_{n n}^{h e} r_{n n}^{e h}=B_{1} B_{2}-r_{n n}^{h e} r_{n n}^{e h}=0, \quad Y=1 .
$$

Therefore, we find that the wave function in the presence of the single impurity at $x=x_{i}$ remains unchanged from the original one

$$
\phi_{n}(x)=\left[\begin{array}{c}
e^{i k x} \\
0
\end{array}\right]+\left[\begin{array}{c}
0 \\
-i e^{-i \varphi} e^{i k x}
\end{array}\right]
$$

The first term represents the incoming wave at the electron branch. The second term expresses the outgoing wave in the hole branch. The Andreev reflection is perfect and the normal reflection is absent even in the presence of the single impurity in the normal metal. With using the
Blonder-Tinkham-Klapwijk formula

$$
G_{\mathrm{NS}}=\frac{e^{2}}{h}\left[1-\left|r_{n n}^{e e}\right|^{2}+\left|r_{n n}^{h e}\right|^{2}\right],
$$

the zero-bias conductance of the NS junction remains unchanged from $G_{\mathrm{NS}}=2 e^{2} / h$ independent of the impurity scattering.

On the way to the conclusion, we derive a relation

$$
r_{n n}^{e h} r_{n n}^{h e}=1
$$

at $E=0$. This plays an important role in the resonant transmission of a quasiparticle in a normal metal. For comparison, the Andreev reflection coefficients of the $s$-wave transparent NS junction become

$$
r_{n n}^{h e}=-i e^{-i \varphi}, \quad r_{n n}^{e h}=-i e^{i \varphi}
$$


at $E=0$. However, they give a relation $r_{n n}^{e h} r_{n n}^{h e}=-1$. In this case, a quasiparticle is scattered by the impurity and the conductance decreases from $G_{\mathrm{NS}}=G_{Q}$. The relation in Eq. (B50) is equivalent to the necessary condition for the formation of Andreev (Majorana) bound states at $E=0$.

\section{APPENDIX C: ANALYSIS OF QUASICLASSICAL USADEL EQUATION}

In this section, we consider a diffusive normal metal is attached to $p_{x}$-wave superconductor as shown in Fig. 5(d). At first, we define the quasiclassical Green's functions in terms of Gor'kov Green's functions. In the mixed representation, Gor'kov Green's functions become

$$
\begin{aligned}
G\left(x, t ; x^{\prime}, t^{\prime}\right) & =G\left(x_{c}, x-x^{\prime}, t_{c}, t-t^{\prime}\right) \\
& =\int \frac{d \epsilon}{2 \pi} \int \frac{d k}{2 \pi} G\left(x_{c}, k, t_{c}, \epsilon\right) e^{i k\left(x-x^{\prime}\right)-i \epsilon\left(t-t^{\prime}\right)}, \\
F\left(x, t ; x^{\prime}, t^{\prime}\right) & =F\left(x_{c}, x-x^{\prime}, t_{c}, t-t^{\prime}\right) \\
& =\int \frac{d \epsilon}{2 \pi} \int \frac{d k}{2 \pi} F\left(x_{c}, k, t_{c}, \epsilon\right) e^{i k\left(x-x^{\prime}\right)-i \epsilon\left(t-t^{\prime}\right)}, \\
x_{c} & =\frac{x+x^{\prime}}{2}, \quad t_{c}=\frac{t+t^{\prime}}{2} .
\end{aligned}
$$

When we consider the static state, the Green's functions are independent of $t_{c}$. With replacing $x_{c}$ by $x$, the quasiclassical Green's functions are defined as

$$
\begin{gathered}
g(x, k, \epsilon)=\frac{i}{\pi} \int d \xi_{k} G(x, k, \epsilon)-\frac{i}{\pi} \int d \xi_{k} \frac{\mathcal{P}}{\xi_{k}}, \\
f(x, k, \epsilon)=\frac{i}{\pi} \int d \xi_{k} F(x, k, \epsilon) .
\end{gathered}
$$

They obey the so-called Eilengerger equation. In what follows, we fix the phase of the superconductor $\varphi$ at 0 . When the normal metal is in the dirty limit, $g(x, k, \epsilon)$ and $f(x, k, \epsilon)$ are isotropic in momentum space. Since they satisfy the normalization condition $g^{2}(x, \epsilon)+f^{2}(x, \epsilon)=1$, it is possible to apply a parametrization: $g(x, \epsilon)=\cos [\theta(x, \epsilon)]$ and $f(x, \epsilon)=$ $\sin [\theta(x, \epsilon)]$. The function $\theta(x, \epsilon)$ obeys the Usadel equation in the diffusive normal metal

$$
D \frac{\partial^{2} \theta(x, \epsilon)}{\partial x^{2}}+2 i \epsilon \sin [\theta(x, \epsilon)]=0,
$$

where $D$ is the diffusion constant in the dirty normal metal.

In what follows, we consider the NS junction shown in Fig. 5(d), where a dirty normal metal is introduced between a clean normal lead wire $(x<-L)$ and a $p_{x}$-wave superconductor $(x>0)$. The boundary conditions for $\theta(x, \epsilon)$ are given by ${ }^{27}$

$$
\theta(x=-L, \epsilon)=0,
$$

$$
\begin{aligned}
\frac{L}{R_{N}} & \left.\frac{\partial \theta(x, \epsilon)}{\partial x}\right|_{x=0} f_{S} \cos \theta_{0}-g_{S} \sin \theta_{0} \\
= & \frac{2}{R_{B}} \frac{\left.t_{n}\right|^{2}+\left|t_{n}\right|^{2}\left(f_{S} \sin \theta_{0}+g_{S} \cos \theta_{0}\right)}{2-\mid t_{n}},
\end{aligned}
$$

with

$$
\begin{gathered}
g_{S}=\frac{g_{+}+g_{-}}{1+g_{+} g_{-}+f_{+} f_{-}}, \quad f_{S}=i \frac{f_{+} g_{-}-f_{-} g_{+}}{1+g_{+} g_{-}+f_{+} f_{-}} \\
R_{B}=\left[G_{Q}\left|t_{n}\right|^{2}\right]^{-1}, \quad G_{Q}=\frac{2 e^{2}}{h}, \quad \theta_{0}=\theta(x=0, \epsilon) .
\end{gathered}
$$

The parameters $R_{N}$ and $R_{B}$ are the normal resistance of the dirty normal metal and that due to the potential barrier at the NS interface, respectively. The information of the pairing symmetry of superconductor is embedded in the surface Green's function $g_{S}$ and $f_{S}$. The total resistance of the junction $R$ is represented by ${ }^{27}$

$$
\begin{gathered}
R=\tilde{R}_{B}+\tilde{R}_{N}, \\
\tilde{R}_{B}=\frac{1}{2} \frac{C_{0}}{\left.\left.\left|\left(2-\left|t_{n}\right|^{2}\right)+\right| t_{n}\right|^{2}\left(\cos \theta_{0} g_{S}+\sin \theta_{0} f_{S}\right)\right|^{2}}, \quad(\mathrm{C} 14) \\
C_{0}=\left|t_{n}\right|^{2}\left(1+\left|\cos \theta_{0}\right|^{2}+\left|\sin \theta_{0}\right|^{2}\right)\left(1+\left|g_{S}\right|^{2}+\left|f_{S}\right|^{2}\right) \\
+4\left(2-\left|t_{n}\right|^{2}\right)\left[\operatorname{Re}\left(g_{S}\right) \operatorname{Re}\left(\cos \theta_{0}\right)+\operatorname{Re}\left(f_{S}\right) \operatorname{Re}\left(\sin \theta_{0}\right)\right] \\
+4\left|t_{n}\right|^{2} \operatorname{Im}\left(\cos \theta_{0} \sin ^{*} \theta_{0}\right) \operatorname{Im}\left(g_{S}^{*} f_{S}\right), \\
\tilde{R}_{N}=\frac{R_{N}}{L} \int_{-L}^{0} \frac{2 d x}{1+|\cos \theta(x, \epsilon)|^{2}+|\sin \theta(x, \epsilon)|^{2}} .
\end{gathered}
$$

The resistances $\tilde{R}_{B}$ and $\tilde{R}_{N}$ are not equal to their normal ones $R_{B}$ and $R_{N}$. They are modified by the proximity effect.

In the case of the $p_{x}$-wave superconductor, the following relations hold:

$$
\begin{gathered}
g_{+}=g_{-}=\frac{\epsilon}{\sqrt{\left(\epsilon+i 0^{+}\right)^{2}-\Delta^{2}}}, \\
f_{+}=-f_{-}=\frac{i \Delta}{\sqrt{\left(\epsilon+i 0^{+}\right)^{2}-\Delta^{2}}} .
\end{gathered}
$$

The $p_{x}$-wave symmetry of superconductor is represented by the relation $f_{+}=-f_{-}$. At the surface of $p_{x}$-wave superconductor, purely odd-frequency pairing states exist due to the formation of the Andreev bound state as discussed in Appendix B.

The Usadel equation can be solved analytically at $\epsilon=0$. Under the boundary condition $\left.\left(L / R_{N}\right)(\partial \theta / \partial x)\right|_{x=0}=i G_{Q}$, we obtain

$$
\begin{gathered}
\theta(x, \epsilon=0)=i R_{N} G_{Q} \frac{x+L}{L}, \\
g(x)=\cos \theta(x, 0)=\cosh \left(R_{N} G_{Q} \frac{x+L}{L}\right), \\
f(x)=\sin \theta(x, 0)=i \sinh \left(R_{N} G_{Q} \frac{x+L}{L}\right)
\end{gathered}
$$

at $\epsilon=0$. The pairing function $f(x)$ represents the spin-triplet $s$-wave odd-frequency pair. Indeed, $f(x)$ is purely imaginary number at $\epsilon=0$. Finally, we obtain the zero-bias resistance

$$
\begin{aligned}
R & =\tilde{R}_{B}+\tilde{R}_{N}=G_{Q}^{-1}, \\
\tilde{R}_{B} & =\frac{1}{G_{Q}}[1+i f(0) / g(0)] \\
& =\frac{1}{G_{Q}}\left[1-\tanh \left(G_{Q} R_{N}\right)\right],
\end{aligned}
$$




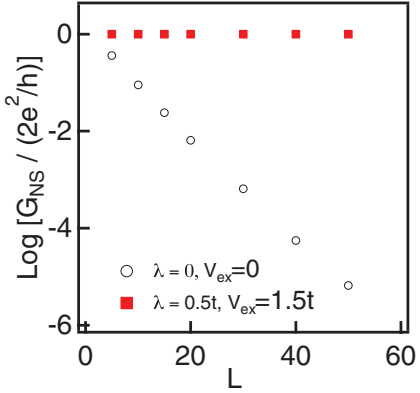

FIG. 6. (Color online) Conductance is plotted as a function of $L$ in NS junctions of nanowire.

$$
\tilde{R}_{N}=\frac{1}{G_{Q}}[-i f(0) / g(0)]=\frac{1}{G_{Q}} \tanh \left(G_{Q} R_{N}\right) .
$$

The total resistance at the zero-bias voltage is independent of $R_{N}$ and $R_{B}$, and remains unchanged from $R=G_{Q}^{-1}$. It is worth to consider the physical meaning of the resulting resistances $\tilde{R}_{B}$ and $\tilde{R}_{N}$. $\tilde{R}_{B}$ is the resistance at the interface which decreases from $G_{Q}^{-1}$ with the increase of $R_{N}$. In other words, the interface resistance decreases with the increase of the amplitude of odd-frequency pair $f(0) . \tilde{R}_{N}$ is the resistance of the dirty normal metal. In the limit of weak proximity effect $R_{N} G_{Q} \ll 1$, the amplitude of odd-frequency pairs becomes small. In such limit, we find $\tilde{R}_{N}=R_{N}$. On the other hand, for $R_{N} G_{Q} \gg 1,-i f(0) / g(0)$ goes to unity and $\tilde{R}_{N}$ approaches to $G_{Q}^{-1}$. Thus, the odd-frequency pairs play a crucial role in the relation of $R=G_{Q}^{-1}$.

The diffusive transport is assumed in the Usadel equation. It is possible to check the validity of $G_{\mathrm{NS}}=G_{Q}$ when the normal segment is in the localization regime. In Fig. 6, we plot the zero-bias conductance in NS junctions of nanowire as a function of the length of the disordered segment $L$. When the nanowire is nontopological at $\lambda=V_{e x}=0$, the zero-bias conductance decreases exponentially with $L$ due to the localization. On the other hand, the conductance for topological nanowire junctions at $\lambda=0.5 t$ and $V_{e x}=$ $1.5 t$ remains unchanged from $G_{Q}$ even in the localization regime.
${ }^{1}$ E. Majorana, Nuovo Cimento 14, 171 (1937).

${ }^{2}$ F. Wilczek, Nat. Phys. 5, 614 (2009); M. Franz, Physics 3, 24 (2010); J. Alicea, Rep. Prog. Phys. 75, 076501 (2012).

${ }^{3}$ D. A. Ivanov, Phys. Rev. Lett. 86, 268 (2001).

${ }^{4}$ S. Das Sarma, M. Freedman, and C. Nayak, Phys. Rev. Lett. 94, 166802 (2005); C. Nayak et al., Rev. Mod. Phys. 80, 1083 (2008).

${ }^{5}$ N. Read and D. Green, Phys. Rev. B 61, 10267 (2000).

${ }^{6}$ C. J. Bolech and E. Demler, Phys. Rev. Lett. 98, 237002 (2007).

${ }^{7}$ K. Sengupta, I. Zutic, H.-J. Kwon, V. M. Yakovenko, and S. Das Sarma, Phys. Rev. B 63, 144531 (2001).

${ }^{8}$ A. Y. Kitaev, Phys. Usp. 44, 131 (2001).

${ }^{9}$ L. Fu and C. L. Kane, Phys. Rev. Lett. 100, 096407 (2008).

${ }^{10}$ M. Sato, Y. Takahashi, and S. Fujimoto, Phys. Rev. Lett. 103, 020401 (2009).

${ }^{11}$ J. D. Sau, R. M. Lutchyn, S. Tewari, and S. Das Sarma, Phys. Rev. Lett. 104, 040502 (2010).

${ }^{12}$ J. Alicea, Phys. Rev. B 81, 125318 (2010).

${ }^{13}$ A. C. Potter and P. A. Lee, Phys. Rev. Lett. 105, 227003 (2010); J. Linder and A. Sudbo, Phys. Rev. B 82, 085314 (2010).

${ }^{14}$ M. Sato and S. Fujimoto, Phys. Rev. B 79, 094504 (2009); Y. Tanaka, T. Yokoyama, A. V. Balatsky, and N. Nagaosa, ibid. 79, 060505 (2009).

${ }^{15}$ S. Sasaki, M. Kriener, K. Segawa, K. Yada, Y. Tanaka, M. Sato, and Y. Ando, Phys. Rev. Lett. 107, 217001 (2011); L. Fu and E. Berg, ibid. 105, 097001 (2010); T. H. Hsieh and L. Fu, ibid. 108, 107005 (2012).

${ }^{16}$ R. M. Lutchyn, J. D. Sau, and S. Das Sarma, Phys. Rev. Lett. 105, 077001 (2010); J. D. Sau, S. Tewari, R. M. Lutchyn, T. D. Stanescu, and S. Das Sarma, Phys. Rev. B 82, 214509 (2010).

${ }^{17}$ Y. Oreg, G. Refael, and F. von Oppen, Phys. Rev. Lett. 105, 177002 (2010).

${ }^{18}$ S. Tewari, T. D. Stanescu, J. D. Sau, and S. Das Sarma, New J. Phys. 13, 065004 (2011); T. D. Stanescu, R. M. Lutchyn, and S. Das Sarma, Phys. Rev. B 84, 144522 (2011); R. M. Lutchyn,
T. D. Stanescu, and S. Das Sarma, Phys. Rev. Lett. 106, 127001 (2011); R. M. Lutchyn and M. P. A. Fisher, Phys. Rev. B 84, 214528 (2011).

${ }^{19}$ A. Golub, I. Kuzmenko, and Y. Avishai, Phys. Rev. Lett. 107, 176802 (2011); K. Flensberg, Phys. Rev. B 82, 180516 (2010); A. Romito, J. Alicea, G. Refael, and F. von Oppen, ibid. 85, 020502 (2012); D. Sticlet, C. Bena, and P. Simon, Phys. Rev. Lett. 108, 096802 (2012); A. C. Potter and P. A. Lee, Phys. Rev. B 83, 094525 (2011); B. Zhou and S.-Q. Shen, ibid. 84, 054532 (2011); K. T. Law and P. A. Lee, ibid. 84, 081304 (2011); J. Klinovaja and D. Loss, ibid. 86, 085408 (2012)

${ }^{20}$ V. Mourik, K. Zuo, S. M. Frolov, S. R. Plissard, E. P. A. M. Bakkers, and L. P. Kouwenhoven, Science 336, 1003 (2012); A. Das, Y. Ronen, Y. Most, Y. Oreg, M. Heiblum, and H. Shtrikman, Nat. Phys. 8, 887 (2012); L. P. Rokhinson, X. Liu, and J. K. Furdyna, ibid. 8, 795 (2012); M. T. Deng, C. L. Yu, G. Y. Huang, M. Larsson, P. Caroff, and H. Q. Xu, Nano Lett. 12, 6414 (2012).

${ }^{21}$ A. R. Akhmerov, J. P. Dahlhaus, F. Hassler, M. Wimmer, and C. W. J. Beenakker, Phys. Rev. Lett. 106, 057001 (2011); P. W. Brouwer, M. Duckheim, A. Romito, and F. von Oppen, Phys. Rev. B 84, 144526 (2011); Phys. Rev. Lett. 107, 196804 (2011); C. Qu, Y. Zhang, L. Mao, and C. Zhang, arXiv:1109.4108; M. Gibertini, F. Taddei, M. Polini, and R. Fazio, Phys. Rev. B 85, 144525 (2012); D. Roy, C. J. Bolech, and N. Shah, ibid. 86, 094503 (2012); M. Wimmer, A. R. Akhmerov, J. P. Dahlhaus, and C. W. J. Beenakker, New J. Phys. 13, 053016 (2011); X.-J. Liu, Phys. Rev. Lett. 109, 106404 (2012); C. H. Lin, J. D. Sau, and S. Das Sarma, Phys. Rev. B 86, 224511 (2012); L.-J. Lang, X. M. Cai, and S. Chen, Phys. Rev. Lett. 108, 220401 (2012); L.-J. Lang and S. Chen, Phys. Rev. B 86, 205135 (2012); F. Pientka, G. Kells, A. Romito, P. W. Brouwer, and F. von Oppen, Phys. Rev. Lett. 109, 227006 (2012); D. Bagrets and A. Altland, ibid. 109, 227005 (2012); A. Zazunov, A. L. Yeyati, and R. Egger, Phys. Rev. B 84, 165440 (2011). 
${ }^{22}$ V. L. Berezinskii, Zh. ETF Pis. Red. 20, 628 (1974) [JETP Lett. 20, 287 (1974)]; A. Balatsky and E. Abrahams, Phys. Rev. B 45, 13125 (1992); T. R. Kirkpatrick and D. Belitz, Phys. Rev. Lett. 66, 1533 (1991); M. Vojta and E. Dagotto, Phys. Rev. B 59, R713 (1999).

${ }^{23}$ F. S. Bergeret, A. F. Volkov, and K. B. Efetov, Phys. Rev. Lett. 86, 4096 (2001); Y. Asano, Y. Tanaka, and A. A. Golubov, ibid. 98, 107002 (2007); V. Braude and Yu. V. Nazarov, ibid. 98, 077003 (2007); Y. Tanaka, A. A. Golubov, S. Kashiwaya, and M. Ueda, ibid. 99, 037005 (2007); Y. Tanaka, Y. Tanuma, and A. A. Golubov, Phys. Rev. B 76, 054522 (2007)

${ }^{24}$ Y. Tanaka, M. Sato, and N. Nagaosa, J. Phys. Soc. Jpn. 81, 011013 (2012).

${ }^{25}$ L. J. Buchholtz and G. Zwicknagl, Phys. Rev. B 23, 5788 (1981); J. Hara and K. Nagai, Prog. Theor. Phys. 74, 1237 (1986); C. R. Hu, Phys. Rev. Lett. 72, 1526 (1994); S. Kashiwaya and Y. Tanaka, Rep. Prog. Phys. 63, 1641 (2000); Y. Tanaka and S. Kashiwaya, Phys. Rev. Lett. 74, 3451 (1995); Phys. Rev. B 56, 892 (1997); Y. Asano, ibid. 64, 014511 (2001); S. Kashiwaya, H. Kashiwaya, H. Kambara, T. Furuta, H. Yaguchi, Y. Tanaka, and Y. Maeno, Phys. Rev. Lett. 107, 077003 (2011).

${ }^{26}$ Y. Tanaka and A. A. Golubov, Phys. Rev. Lett. 98, 037003 (2007).

${ }^{27}$ Y. Tanaka, Y. Asano, A. A. Golubov, and S. Kashiwaya, Phys. Rev. B 72, 140503(R) (2005); Y. Tanaka and S. Kashiwaya, ibid. 70, 012507 (2004); Y. Tanaka, S. Kashiwaya, and T. Yokoyama, ibid. 71, 094513 (2005).
${ }^{28}$ Y. Tanaka, Yu. V. Nazarov, and S. Kashiwaya, Phys. Rev. Lett. 90, 167003 (2003)

${ }^{29}$ Y. Asano, Y. Tanaka, and S. Kashiwaya, Phys. Rev. Lett. 96, 097007 (2006).

${ }^{30}$ Y. Asano, Y. Tanaka, A. A. Golubov, and S. Kashiwaya, Phys. Rev. Lett. 99, 067005 (2007).

${ }^{31}$ Y. Asano, A. A. Golubov, Y. V. Fominov, and Y. Tanaka, Phys. Rev. Lett. 107, 087001 (2011).

${ }^{32}$ Recent papers have shown that topological superconductivity is stable for weak interactions, S. Gangadharaiah, B. Braunecker, P. Simon, and D. Loss, Phys. Rev. Lett. 107, 036801 (2011); E. M. Stoudenmire, J. Alicea, O. A. Starykh, and M. P. A. Fisher, Phys. Rev. B 84, 014503 (2011).

${ }^{33}$ P. A. Lee and D. S. Fisher, Phys. Rev. Lett. 47, 882 (1981).

${ }^{34}$ The normal conductance $g_{\mathrm{N}}$ in units of $2 e^{2} / h$ well obeys a relation of $\ln \left(g_{\mathrm{N}}\right) \propto-L / \xi_{\mathrm{AL}}$ with $\xi_{\mathrm{AL}}$ with being the localization length. We estimate that $\xi_{\mathrm{AL}}$ for the topological $\left(V_{e x}=\lambda=0\right)$ and the nontopological $\left(V_{e x}=1.5 t, \lambda=0.5 t\right)$ nanowires are about 11 and 8.5 lattice constants, respectively.

${ }^{35}$ G. E. Blonder, M. Tinkham, and T. M. Klapwijk, Phys. Rev. B 25, 4515 (1982).

${ }^{36}$ Y. Asano, Y. Tanaka, and S. Kashiwaya, Phys. Rev. B 69, 214509 (2004).

${ }^{37}$ J. D. Sau, B. I. Halperin, K. Flensberg, and S. Das Sarma, Phys. Rev. B 84, 144509 (2011). 